\title{
Capacité, vieillissement de la population et professionnalisme
}

$\mathrm{L}$ 'intervention du médecin pour atténuer la souffrance et améliorer les fonctions ne constitue que l'un des éléments de la relation médecin-patient. Pour intervenir avec bienveillance (et ne causer aucun tort), il faut agir en partenariat avec les patients. Les médecins recherchent ce partenariat parce qu'ils reconnaissent l'autonomie des patients et parce que sans lui, ils risquent de faire preuve de paternalisme. Nous pouvons facilement accepter la primauté de l'autonomie lorsque des patients capables de s'exprimer échangent activement avec nous, mais cela devient plus difficile lorsque leur capacité est compromise. En rattachant étroitement le respect de l'autonomie à des mécanismes liés au consentement légal, nous avons oublié notre obligation de ne pas adopter pour traiter les patients des pratiques et des milieux physiques qui minent leur capacité.

En théorie, la capacité est évaluée et modifiable. En pratique, il faut pouvoir la cerner et la réévaluer, et prévoir la prise de décision par des tiers. Dans la pratique médicale quotidienne, et en particulier dans les hôpitaux ${ }^{1}$, les pressions du temps, la complexité des besoins des patients et la perception du consentement comme intrinsèque - dans une population qui, en grande partie, donne effectivement son consentement - ont pour résultat que le respect de l'autonomie est souvent a posteriori, théorique et symbolique.

Deux raisons m'incitent à parler de la façon dont ignore largement l'autonomie des personnes âgées frêles (aux prises avec de multiples problèmes médicaux et sociaux interreliés) en contexte hospitalier de soins actifs. Beaucoup de personnes âgées frêles sont de toute évidence en possession de leurs moyens lorsqu'elles ne sont pas malades et qu'elles ont une routine structurée dans un environnement qu'elles connaissent bien. Lorsqu'elles tombent malades et sont hospitalisées, leur capacité peut toutefois être compromise rapidement, même en l'absence de délire véritable. Comme les aînés frêles sont proportionnellement les plus grands consommateurs de soins de santé actifs, nous devons soit trouver un moyen de structurer et de faciliter le mécanisme de consentement, soit mieux comprendre si nos actions, dans le contexte habituel des soins, se justifient sur le plan de l'éthique. Il nous faut aussi examiner attentivement notre mode actuel de fonctionnement, car si nous ne séparons pas la pratique réelle de la théorie d'une pratique idéale, nous n'aurons aucun moyen de distinguer une bonne pratique d'une mauvaise : chacune sera plutôt condamnée à l'échec parce qu'elle ne respectera pas idéalement l'autonomie.

Je ne préconise pas de recourir davantage au consentement éclairé, même si certains affirment que ce serait à la fois souhaitable et faisable ${ }^{2}$. Je pense que nous devons tenir les médecins et les hôpitaux responsables, non parce qu'ils ont supposé l'existence du consentement au lieu de recourir aux procédures nécessaires pour l'obtenir, mais plutôt parce qu'ils ont supposé que les intéressés consentaient à des actes offrant peu d'espoir d'atténuer la souffrance, d'améliorer leurs fonctions ou in d'éviter une mort évitable. Chez les personnes âgées frêles, je constate plus de traumatismes causés par le fait que l'on n'accorde pas suffisamment d'attention à nos obligations de ne causer aucun préjudice et de faire du bien que par le défaut d'obtenir le consentement. L'exemple le plus évident est celui du patient âgé qui a donné son plein consentement et dont la hernie, le cœur ou la hanche ont guéri après une chirurgie élective, mais dont la cogni- tion et le fonctionnement ont subi un préjudice irréparable parce qu'on a omis d'évaluer sa fragilité avant l'intervention.

C'est pourquoi je crois que nous devons explorer la façon dont l'autonomie des patients âgés frêles dépend étroitement de leur milieu et de leur état de santé. Ces connaissances nous permettront de respecter leur autonomie en concevant des environnements et des plans de soins (y compris l'évaluation de routine de la fragilité) qui amélioreront la capacité au lieu de la compromettre. Cette analyse pourrait s'étendre aux innovations qui nous aideraient à fournir aux patients des soins à domicile, là où leur autonomie risque le moins d'être compromise ${ }^{3}$.

Nous devons aussi mieux comprendre comment les médecins devraient interagir avec les patients âgés frêles dont la compétence est manifestement incertaine. La bienfaisance et la nonmalveillance ne sont-elles que des relents du bon vieux paternalisme? Les médecins peuvent-ils trouver une façon de respecter l'éthique en travaillant avec des patients frêles, sans que ceux-ci aient à faire valoir leur autonomie et sans écarter leur capacité diminuée? La solution pourrait résider dans un renouvellement $\mathrm{du}$ professionnalisme, la reconnaissance du fait que les médecins sont tenus d'utiliser leurs connaissances, leurs compétences cliniques et leur jugement pour le bénéfice de leurs patients. Un groupe du Royaume-Uni a recommandé que chaque médecin réfléchisse à la définition du professionnalisme médical et à son pouvoir discrétionnaire afin de déterminer comment il l'exprime dans sa pratique quotidienne $e^{4}$. Pourrait-il en découler une théorie du professionnalisme qui coïnciderait davantage avec les limites de l'autonomie de nos patients frêles? Le cas échéant, cela permettrait de distinguer la bonne pratique de la mauvaise ${ }^{5,6}$.

On sait depuis Hippocrate que le professionnalisme est chèrement gagné : «La vie est courte, l'art, long, l'occasion, éphémère, l'expérience, fallacieuse, et le jugement, difficile.» Si nous voulons fournir de meilleurs soins à nos patients âgés frêles, nous devons réfléchir attentivement à la façon de baser la relation médecinpatient sur une confiance bien fondée et l'améliorer.

\section{Kenneth Rockwood}

Professeur de médecine (gériatrie et neurologie)

Université Dalhousie

Halifax (N.-É.)

Cet article a fait l'objet d'un examen par les pairs.

\section{RÉFÉRENCES}

I. Raymont V, Bingley W, Buchanan A, et al. Prevalence of mental incapacity in medical inpatients and associated risk factors: cross-sectional study. Lancet 2004;364:I42I-7. 2. Karlawish JH. Competency in the age of assessment. Lancet 2004;364:1383-4.

3. Caplan GA, Coconis J, Board N, et al. Does home treatment affect delirium? A randomised controlled trial of rehabilitation of elderly and care at home or usual treatment (the REACH-OUT trial). Age Ageing 2006;35:53-6o.

4. Tallis RC. Doctors in society: medical professionalism in a changing world. Clin Med 2006;6:7-I2.

5. Tauber AI. Patient autonomy and the ethics of responsibility. Cambridge (MA) : MIT Press; 2005

6. Agich GJ. Dependence and autonomy in old age: an ethical framework for longterm care. Cambridge (R. U.) : Cambridge University Press; 2003. 